Med Klin Intensivmed Notfmed 2014 .

109:226-227

DOI 10.1007/s00063-013-0302-7

Online publiziert: 27. April 2014

(c) Springer-Verlag Berlin Heidelberg 2014

\section{Bruno Schneewei $\aleph^{1} \cdot$ Ingrid Pretsch ${ }^{2}$}

${ }^{1}$ Interne Abteilung, LKH Kirchdorf

2 Universitätsklinik für Innere Medizin II, Kardiologie und internistische Intensivmedizin,

Universitätsklinikum der Paracelsus Medizinischen Privatuniversität, Landeskrankenhaus Salzburg

\title{
Die Kunst der Behandlung des Multiorganversagens
}

Liebe Kolleginnen und Kollegen,

Unter dem Motto „Die Kunst der Behandlung des Multiorganversagens" findet die diesjährige 46. gemeinsame Jahrestagung der Österreichischen Gesellschaft für Internistische und Allgemeine Intensivmedizin und Notfallmedizin (ÖGIAIN) und der Deutschen Gesellschaft für Internistische Intensivmedizin und Notfallmedizin (DGIIN) in Salzburg statt.

In den letzten Jahren hat sich das Spektrum der Krankheitsbilder, die an Intensivstationen behandelt werden, gewandelt: Das durch unterschiedliche Ursachen bedingte Mehrorganversagen stellt weiterhin die größte Herausforderung an den Intensivmediziner dar.

I) Das Mehrorganversagen stellt die größte Herausforderung dar

In der vorliegenden Ausgabe der Zeitschrift Medizinische Klinik - Intensivmedizin und Notfallmedizin werden an Hand von 5 Beiträgen ausgewiesener Experten einige pathophysiologisch und klinisch relevante Aspekte der Organinteraktionen bei diesem schweren Krankheitsbild aufgezeigt.

In 4 Arbeiten werden die zentrale Rolle der Leber im Multiorganversagen und ihre Interaktion mit anderen Organen aufgezeigt. Eine Störung der Leberfunktion im Sinne einer Schockleber und cholestatischen Dysfunktion wird bei 10$30 \%$ der kritisch kranken Patienten beobachtet, wobei neben iatrogenen Faktoren besonders hypoxische Ereignisse, wie
Sepsis und zirkulatorisches Versagen unterschiedlicher Ätiologie, als auslösende Ursache zugrunde liegen.

Der Einfluss einer gestörten Leberfunktion auf die Herz- und Lungenfunktion kann sich als hepatopulmonales Syndrom (HPS), portopulmonale Hypertension (POPH) und als hepatischer Hydrothorax manifestieren. Das HPS tritt bei bis zu 30\% der Patienten mit Leberzirrhose auf und ist mit einer deutlich gesteigerten Mortalität verbunden, da auch die medikamentösen therapeutischen Möglichkeiten eingeschränkt sind. Beim POPH können unterschiedliche Vasodilatoren eine Senkung des pulmonalarteriellen Drucks bewirken und so den Krankheitsverlauf günstig beeinflussen.

Eine Nierenfunktionseinschränkung wird bei etwa $20 \%$ der Patienten mit fortgeschrittener Leberzirrhose beobachtet, wobei prärenale und intrarenale Faktoren ursächlich verantwortlich sind. Ist die Nierenfunktionseinschränkung durch eine Abnahme des effektiven Blutvolumens bedingt, spricht man von einem hepatorenalen Syndrom (HRS). Therapeutisch kann durch die Gabe von Vasopressoren in Kombination mit Humanalbumin bei etwa 50\% der Patienten das HRS erfolgreich behandelt werde.

Das häufige Auftreten eines Multiorganversagens bei akuten und chronischen Lebererkrankungen bedingt auch häufig den Einsatz extrakorporaler Therapieverfahren. In einem Artikel dieses Themenhefts wird deshalb die derzeitige Praxis des in seiner Bedeutung für das Überleben der Patienten noch ungesicherten extrakorporalen Lebersupports diskutiert. 
Renale Dysfunktionen werden auch im Verlaufe einer kardialen Dysfunktion häufig beobachtet und traditionell auf ein Vorwärtsversagen zurückgeführt.

Das Ausmaß der renalen Dysfunktion scheint aber eher mit Indikatoren des gestörten venösen Rückflusses (Stauung) zu korrelieren, der durch eine Reihe von Organdysfunktionen, z. B. des rechten Herzes, der Lunge und Leber und auch des Darms mit seinen proinflammatorischen Signalen, beeinflusst wird. Ganz besonders an diesem kardiopulmonalen Syndrom werden das Zusammenspiel der einzelnen Organe und ihre Störung im Multiorganversagen sichtbar.

Wir hoffen, mit diesen Beiträgen zur Pathophysiologie und Therapie des Multiorganversagens einen repräsentativen Überblick zum Leitthema der 46. Jahrestagung der ÖGIAIN und DGIIN zu bieten und würden uns freuen, Sie im Juni 2014 in Salzburg begrüßen zu dürfen.

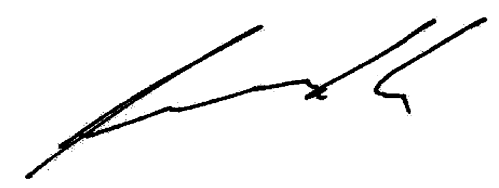

Univ.-Prof. Dr. Bruno Schneeweiß

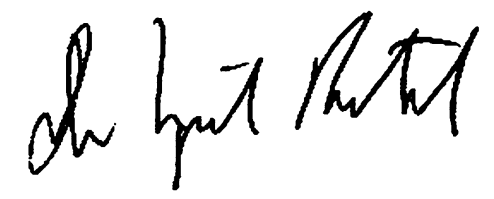

OA Dr. Ingrid Pretsch

\section{Korrespondenzadresse}

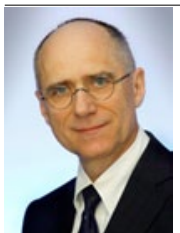

Univ.-Prof.

Dr. Bruno Schneeweiß

Interne Abteilung,

LKH Kirchdorf

Hausmanningerstraße 8,

A-4560 Kirchdorf

Österreich

bruno.schneeweiss@gespag.at

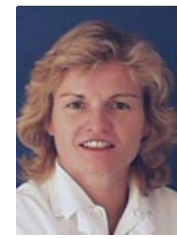

OA Dr. Ingrid Pretsch

Universitätsklinik für Innere

Medizin II, Kardiologie und

internistische Intensivmedizin,

Universitätsklinikum der

Paracelsus Medizinischen

Privatuniversität,

Landeskrankenhaus Salzburg

Müllner Hauptstraße 48,

A-5020 Salzburg

Österreich

i.pretsch@salk.at

Interessenkonflikt. B. Schneeweiß und I. Pretsch geben an, dass kein Interessenkonflikt besteht.

\section{Leipziger Studie zu Langzeit- folgen von Krebs}

In Deutschland leben über 2 Millionen Menschen, bei denen eine Krebsdiagnose bis zu zehn Jahre zurückliegt. Die Spät- und Langzeitfolgen einer Krebserkrankung führen zu neuen Herausforderungen in der Gesundheitsversorgung und in der Rehabilitation. Nun startet ein dreijähriges Forschungsprojekt, das die körperlichen und psychischen Spät- und Langzeitfolgen von Krebserkrankungen erfasst. Dazu sollen etwa 800 Menschen befragt werden, bei denen die Krebsdiagnose 5 bzw. 10 Jahre zurückliegt.

„Bislang wissen wir noch zu wenig über die körperlichen und psychosozialen Langzeit- und Spätfolgen der Erkrankung und in welchem Ausmaß diese den Alltag der Patienten beeinträchtigen", sagt Prof. Anja Mehnert, Leiterin der Sektion Psychosoziale Onkologie an der Abteilung Medizinische Psychologie und Medizinische Soziologie der Universität Leipzig.

Die Wissenschaftler interessiert, welche onkologischen und psychoonkologischen Versorgungsangebote Krebspatienten 5 bzw. 10 Jahre nach der Akutbehandlung in Anspruch nehmen und wie zufrieden sie damit sind. "Wir wollen herausfinden, wodurch die seelische und körperliche Gesundheit nach einer Krebserkrankung gestärkt werden kann", erklärt die Projektleiterin Dr. Heide Götze.

Das Projekt wird gefördert durch die Stiftung Swiss Bridge. Die Stiftung unterstützt Projekte in der Onkologie, die darauf abzielen, die medizinische Versorgung von Krebspatienten zu verbessern.

Quelle:

Universität Leipzig, www.uni-leipzig.de 